\title{
SUMMARY OF AVAILABLE AND PLANNED ATOMIC DATABASES
}

\author{
W. C. MARTIN \\ Atomic Energy Levels Data Center \\ National Institute of Standards and Technology \\ Gaithersburg, MD 20899 \\ U.S.A.
}

Several of the larger and more inclusive atomic data centers and databases useful for space astronomy are briefly described here. Bibliographic reports on atomic and molecular data useful for astronomy are given in Ref. [1].

\section{Energy Levels, Wavelengths, Transition Probabilities, and Line Broadening}

The Atomic Energy Levels Data Center and the Data Center on Atomic Transition Probabilities at the National Institute of Standards and Technology (NIST) compile and publish critically evaluated data on atomic energy levels, wavelengths, and transition probabilities. These Centers are also building an atomic spectroscopic database for astronomy, which now includes evaluated data for most spectra of the elements hydrogen through nickel $(\mathrm{Z}=1-28)$ and for many spectra of the heavier elements (see, e.g., $[2-4])$.

New transition probability data for spectra of the first ten elements now being compiled and entered into the NIST database by W. L. Wiese $e t$ al. originate mainly from the Opacity Project [5]. It is planned that data from the Opacity Project will be maintained in databases in several locations, including the Dept. of Astronomy at Ohio State University, Columbus, $\mathrm{OH}$, and the National Center for Supercomputing Applications at the University of Illinois, Urbana (A. Pradhan); the Database on Atomic and Molecular Physics at the Queen's University of Belfast (K. A. Berrington, F. J. Smith); and the Scientific Center at IBM de Venezuela, Caracas (C. Mendoza).

R. L. Kurucz of the Harvard-Smithsonian Center for Astrophysics has calculated and compiled spectroscopic data for stellar atmospheric modeling [6]. His data on wavelengths, gf values, and damping constants include experimental or experimentally based wavelengths for 555,000 atomic lines. Some 410,000 of these lines belong to the first through the ninth (I through IX) spectra of the iron-group elements calcium through nickel; Kurucz has calculated data for $\mathbf{4 2}$ million lines of these elements.

A new bibliographic database on atomic spectral line shapes and shifts will be maintained jointly by the Département d'Astrophysique Stellaire et Galactique of the Observatoire de Paris-Meudon (A. Lesage) and by the NIST Data Center on Atomic Line Shapes and Shifts (J. Fuhr). Their plans include publication of annotated bibliographies. 


\section{Photoionization and Collisions}

New photoionization data have been calculated for atoms and ions of the first ten elements as a part of the Opacity Project [5].

In addition to photoexcitation and photoionization, the Belfast group has emphasized electron impact excitation data for atoms and ions of astrophysical interest. The Atomic Collisions Database at the Joint Inst. for Laboratory Astrophysics, Boulder, CO, also has extensive data of these types (J. Broad).

Heavy Particle collisions are emphasized by the Controlled Fusion Atomic Data Center in Oak Ridge, TN (R. A. Phaneuf), and by two Japanese centers: the Data and Planning Center of the National Inst. of Fusion Science, Nagoya (H. Tawara), and the Atomic and Molecular Data Unit of the Japan Atomic Energy Research Inst., Tokai-mura (T. Shirai).

\section{All Types of Atomic Data}

The GAPHYOR Data Center, Université Paris-Sud, Orsay, has extensive bibliographic files covering the types of data mentioned above and also data on clusters and interactions of atoms and molecules with solids (J.-L. Delcroix).

The Atomic and Molecular Data Center Network of the International Atomic Energy Agency (IAEA, Vienna) comprises 15 data centers, including most of those mentioned above. An increasing amount of data supplied by various Network centers is maintained in a uniform format by the Atomic and Molecular Data Unit of the IAEA. This Unit also issues a useful bibliographic bulletin twice yearly [7] (R. K. Janev).

\section{References}

[1] McNally, D. (Ed.) 1991, Reports on Astronomy: Trans. IA.U. XXI A, Kluwer Academic, Dordrecht, pp. 105-136.

[2] Sugar, J. and Corliss, C. 1985, Atomic Energy Levels of the Iron-Period Elements: Potassium through Nickel, J. Phys. Chem. Ref. Data 14, Suppl. 2.

[3] Martin, G. A., Fuhr, J. R. and Wiese, W. L. 1988, Atomic Transition Probabilities-Scandium through Manganese, J. Phys. Chem. Ref. Data 17, Suppl. 3.

[4] Fuhr, J. R., Martin, G. A. and Wiese, W. L. 1988, Atomic Transition Probabilities-Iron through Nickel, J. Phys. Chem. Ref. Data 17, Suppl. 4.

[5] Seaton, M. J. 1987, J. Phys. B 20, 6363-6378; Mendoza, C., summary of OpacityProject results in this volume.

[6] Kurucz, R. L. 1991, "New Opacity Calculations", in Crivellari, L., Hubeny, I. and Hummer, D. G. (Eds.), Stellar Atmospheres: Beyond Classical Models, NATO ASI Series, Kluwer Academic, Dordrecht, pp. 440-448.

[7] Smith, J. J. (Ed.) 1991, International Bull. Atomic and Molec. Data for Fusion, No. 42, Int. Atomic Energy Agency, Vienna; and preceding issues. 